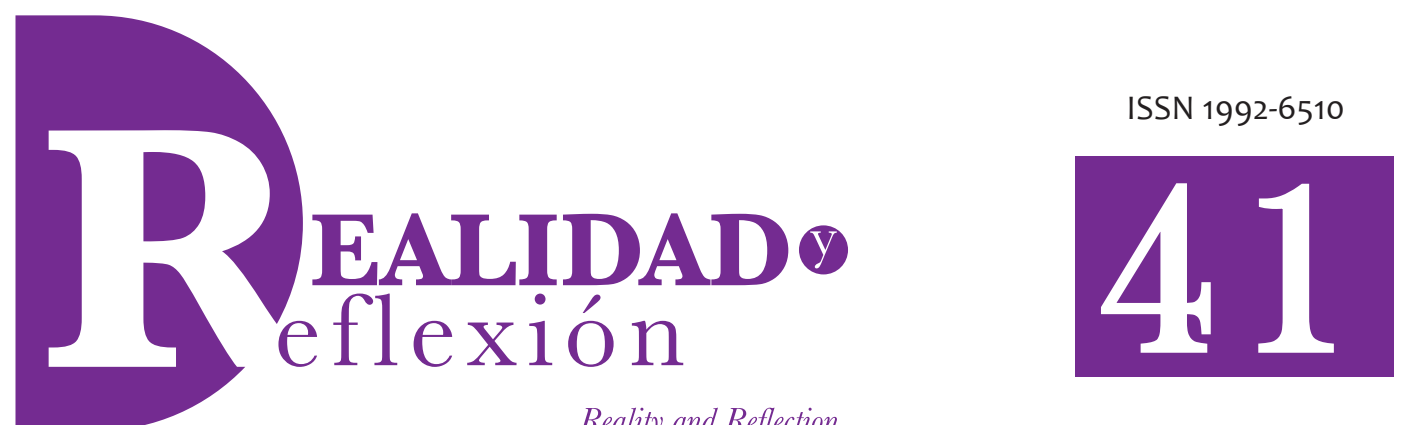

Reality and Reflection

año 15, N 41, San Salvador, El Salvador, Centroamérica Revista Semestral Enero-Junio 2015

YeAR 15, N41, San Salvador, El Salvador, Central America Semestral Journal January-June 2015

\title{
Evaluación de impacto en los programas de formación profesional en El Salvador
}

\section{Evaluation of the impact on the vocational training programs in El Salvador}

\author{
Ricardo Olmos Guevara \\ B.A en Economía, \\ Universidad del Quebec en Montreal, Canadá. \\ Licenciatura en Economía, \\ Universidad Centroamericana, José Simeón Cañas. \\ M.A. en Finanzas, \\ Universidad de El Salvador. \\ Gerente de Política y Planificación de Transporte, \\ Viceministerio de Transporte. \\ rholmos@hotmail.com
}

\section{RESUMEN}

El autor presenta los resultados de un estudio de impacto de los programa de formación profesional en El Salvador. Estos indican que los cursos de actualización y formación profesional son vitales para incidir en el bienestar del participante y en el de su familia, dado que se eleva la condición de empleabilidad y mejora sustantivamente los ingresos. El autor aplica las herramientas econométricas para determinar los impactos en la formación profesional en materia de empleo y de ingreso, utilizando para tal fin el enfoque econométrico de la doble diferencia.

FORMACIÓN PROFESIONAL, COMPETENCIA PROFESIONAL, EL SALVADOR- FORMACIÓN PROFESIONAL.

\section{ABSTRACT}

The author presents the results of an impact study of the vocational training program in El Salvador. The results indicate that refresher courses and training are vital to influence the welfare of the participant and his family since employability status rises and significantly improves revenues. The author applies the econometric tools to determine the impacts on professional training in employment and income for this purpose using the econometric approach of double difference. PROFESSIONAL TRAINING, PROFESSIONAL COMPETENCE, EL SALVADOR TRAINING. 


\section{Introducción ${ }^{1}$}

El objetivo central de este estudio es evaluar los impactos de la oferta formativa que se extiende a través de INSAFORP (Instituto Salvadoreño de Formación Profesional), con el Programa Hábil Técnico Permanente, PHTP, que se ofreció durante los años 2010-2011 a la población desempleada y subempleada, así como a otros grupos poblacionales que demandan capacitación técnica.

El estudio se centra en la medición de la efectividad y la cobertura geográfica del Programa de formación para atender a la población beneficiaria en sus distintas condiciones socioeconómicas, y posibilitar una oferta más amplia en diferentes cursos técnicos; así como disponer de una oferta formativa flexible en cuanto a duración, horario y número de participantes, con la convicción y la seguridad de lograr una ejecución sostenida o permanente con la mayor pertinencia en temas de capacitación, lo que se vuelve estratégica para aquellas instituciones que realizan política social, como lo es INSAFORP.

La evaluación del Programa pretende medir si efectivamente las necesidades de los participantes, de acuerdo a sus propias expectativas de inserción productiva y en el marco de la política social del país, se unen para propiciar y fomentar la igualdad de oportunidades de capacitación para mujeres y hombres que buscan mejorar su empleabilidad. Finalmente, los resultados que se presentan en este estudio de evaluación de impacto buscan, además,

1 Este estudio es la síntesis de una consultoría realizada para INSAFORP, efectuada del 31 de julio de 2013 hasta enero de 2014, en el marco de la Evaluación del Programa de Formación Profesional. Para ello se realizó un levantamiento de la información de la muestra seleccionada para tal fin. medir los impactos generados y evidenciar la mejora de las condiciones de vida de las personas beneficiarias de la capacitación.

Los resultados indican que la profundización en la mejora en la formación del capital humano de los participantes en los programas que impulsa INSAFORP tienden en el corto plazo a mejorar las competencias técnicas, y en definitiva, a optimizar la productividad del trabajo con modalidades diferentes de formación y con un menú de áreas de formación que satisfacen la demanda orientada a lo que el país requiere y que su aparato productivo demanda.

$Y$ en efecto, los aspectos que impactan directamente la empleabilidad están referidos a las oportunidades de formación profesional en cada una de las áreas de formación que forman parte de la oferta en el PHTP. El nivel socioeconómico del cual provienen, el género, el nivel educativo, la situación de la condición familiar, y la zona de residencia son algunos de los elementos que, como otros, pesan en el proceso de inserción laboral.

Esos aspectos están a la base de las condiciones elementales de un determinando impacto (Gallart, 2003) En América Latina, son importantes las instituciones vinculadas con la formación para desempleados, o que no obstante, tengan un empleo y desean actualizarse, lo que constituye parte del paradigma, el cual se encuentra a la base de la formación laboral y que se establece para determinados sectores sociales, como el instrumento más apropiado para reducir las probabilidades de caer y mantenerse en condiciones de pobreza en espacios intergeneracionales amplios. 
El estudio realizado se ejecutó con un marco muestral de 30,177 participantes, para los cuales se aplicó el enfoque econométrico de diferencia en la diferencia para observar los impactos socioeconómicos pretendidos.

En este estudio se observa a nivel nacional el impacto global en las 19 especialidades, y que fue medido a través de la muestra seleccionada de 390 participantes. La inserción productiva observada procede desde aquellas oportunidades en los nichos ocupacionales del mercado, que son precisamente aquellos en los que el aprendizaje en el trabajo y el valor de la experiencia es menor (INSAFORP, 20012002). Y de esa manera se transita hacia las ocupaciones a tiempo completo con las mayores prestaciones sociales, y que según la OIT lo denomina empleo decente.

\section{Aplicación de Modelo de diferencia en diferencia}

\subsection{Diferencias en diferencias del Programa} Hábil Técnico Permanente: el caso del empleo

El método de diferencia en diferencia nos permite conocer los aspectos de impactos del Programa frente a los grupos de comparación. Este método consiste, como indica su nombre, en aplicar una doble diferencia. Compara los cambios a lo largo del tiempo en la variable de interés entre una población inscrita en un programa (grupo de tratamiento) y una población no inscrita (el grupo de comparación), (Billorou). En el caso de este estudio los impactos son más que evidentes, pues la primera diferencia indica que efectivamente los participantes han aprovechado los cursos de formación profesional, pues una vez obtenida la formación, su actitud hacia el empleo es más que evidente, y que se ha destacado en el capítulo anterior. Ello ha generado inserción productiva y, por supuesto, mayor incidencia en su bienestar personal y de su familia (Cuadro 1).

La figura $\mathrm{N}^{\circ} 1$ establece la relación funcional entre aquellos participantes que alcanzaron y sobrepasaron a los que efectivamente no asistieron a ningún curso de formación profesional. El grupo de comparación fue seleccionado con las mismas características del grupo que sufrió el tratamiento. La medición del impacto del grupo de tratamiento se evidencia frente al grupo de comparación en lo que respecta al empleo de aquellos que, luego de los cursos, lograron aplicar en el mercado laboral.

\section{Figura 1}

\section{El Salvador. Evaluación de impacto del empleo del PHTP.}

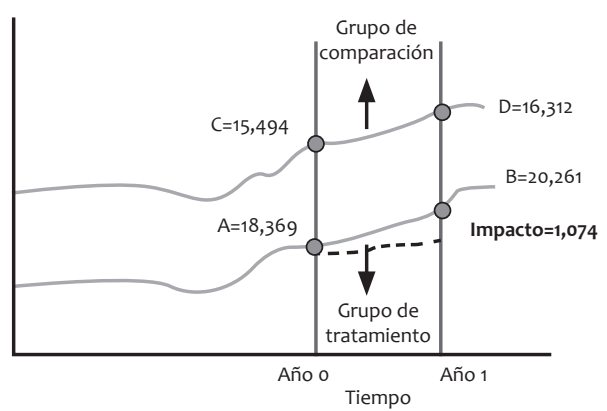

Fuente: Elaboración propia.

El resultado de la diferencia entre el grupo de tratamiento y grupo de comparación indica que la velocidad de incremento del empleo en el grupo de tratamiento es superior en 1,074 puntos versus el grupo de comparación.

Otro tipo de mediciones realizadas en el levantamiento de información, realizado 


\section{Cuadro 1}

El Salvador. Impacto del Programa Hábil Técnico Permanente, PHTP según el Método de la diferencia en diferencias en materia de empleo entre los grupos de tratamiento y grupo de comparación.

\begin{tabular}{lccc}
\hline & Después & Antes & \\
\hline Grupo de tratamiento & B & A & B - A \\
\hline Grupo de comparación & D & C & D - C \\
\hline Diferencia & B - D & A - C & DD = (B - A) - (D - C) \\
\hline Después & Antes & 1,892 \\
\hline Grupo de tratamiento & 20,261 & 18,369 & 818 \\
\hline Diferencia & 16,312 & 15,494 & 1,074 \\
\hline
\end{tabular}

Fuente: Banco mundial 2013 y elaboración propia.

en ambos grupos de estudio para cada una de las ramas del aparato productivo salvadoreño, indicó que los participantes del PHTP incrementaron su inserción laboral en 10.3\%; mientras que el grupo de comparación lo hizo con un $5.2 \%$ de incremento en el mercado laboral. El PHTP posee un elevado impacto por encima del grupo de comparación.

\subsection{Diferencias en diferencias del Programa} Hábil Técnico Permanente: el caso de los ingresos

La evaluación realizada tomando en consideración la variable ingresos asegura que el grupo de tratamiento frente al grupo de comparación es en 9.6 puntos, con lo que se puede indicar que en efecto existe un impacto acelerador en la vida de los participantes con los cursos de formación de INSAFORP.

Cuando se observa solamente la evolución que en el tiempo registran aquellos que no se han vinculado a ningún curso de formación, los resultados de ese grupo contrafactual de comparación fueron de solamente 8.4 puntos, lo que indica que en términos generales los efectos de los que no se inscribieron han tenido un impacto menor con respecto al grupo que recibió tratamiento. Mientras aquellos que efectivamente recibieron el tratamiento, es decir, los cursos de formación, se colocan con 18.0 puntos en su evolución a través del tiempo.

Cuando se realiza la doble diferencia se tiene un resultado favorable, pues se tienen impactos globales positivos que suman 9.6 puntos (Cuadro 2 ).

En la figura $\mathrm{N}^{\circ} 2$ se evidencia el resultado de la diferencia entre el grupo de tratamiento y el grupo de comparación en términos de la velocidad de incremento de los ingresos en el grupo de tratamiento el cual es superior en 9.6 puntos versus el grupo de comparación. 


\section{Cuadro 2}

El Salvador. Resultados de la aplicación del Método de la diferencia en diferencia sobre los ingresos en el Programa Hábil Técnico Permanente, PHTP.

\begin{tabular}{lcccc}
\hline & Después & Antes & \\
\hline Grupo de tratamiento & B & A & B - A \\
\hline Grupo de comparación & D & C & D - C \\
\hline Diferencia & B - D & A - C & DD = (B - A ) - (D - C) \\
\hline & Después & Antes & 18 \\
\hline Grupo de tratamiento & 303 & 285 & 8.4 \\
\hline Grupo de comparación & 348.7 & 340.7 & 9.6 \\
\hline Diferencia & -45.7 & -55.3 & \\
\hline
\end{tabular}

Fuente: Banco mundial 2013 y elaboración propia.

Figura 2

El Salvador. Evaluación de impacto del ingreso del Programa Hábil Técnico Permanente

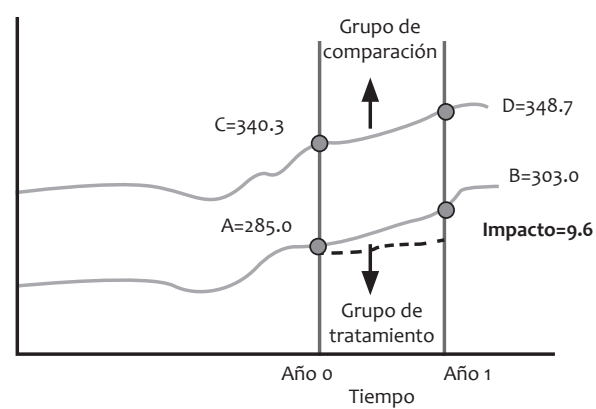

Fuente: Elaboración propia.

En otro tipo de mediciones realizado a través del levantamiento de información, como es el volumen de ingresos y su variación a lo largo del tiempo en cada una de las ramas del aparato productivo, en donde se localizan tanto el grupo de comparación como el grupo de tratamiento, la investigación efectuada indica que existe la variación de los ingresos del grupo de comparación, con 2.3\%; mientras que la variación del grupo de tratamiento es de 6.3\%. Eso implica que el impacto de los cursos de formación generó un impacto positivo en los ingresos de los participantes.

En síntesis, el estudio presenta resultados favorables para aquellos participantes en este PHTP, pues en cada uno de los aspectos considerados de empleo e ingresos, experimentaron que en efecto hay un impacto sumamente fuerte y por encima de los que no se someten a este tipo de iniciativas de formación.

El método de diferencias en diferencias computa la estimación del impacto de la siguiente manera:

1. Se calcula la diferencia del resultado (Y) entre las situaciones antes y después para el grupo de tratamiento (B-A).

2. Se calcula la diferencia del resultado (Y) entre las situaciones antes y después para el grupo de comparación (D-C).

3. A continuación se calcula la diferencia entre la diferencia en los resultados del 
grupo de tratamiento (B-A) y la diferencia del grupo de comparación ( $D-C)$, o DD $=(B$ A) - (D-C). Esta diferencias en diferencias es la estimación de impacto (Billorou).

\section{Aplicación econométrica del modelo de diferencias en diferencias}

\subsection{Diferencias en diferencias del Programa Hábil Técnico Permanente: Enfoque econométrico}

Los estudios cuasi experimentales intentan evaluar la efectividad de una intervención comparando dos grupos, uno de los cuales la recibe y otro no. En tal sentido, en este estudio se aborda y se da respuesta a la interrogante ¿Cuál es la situación de los participantes luego de haberse ejecutado el proyecto en sus diferentes acciones de formación?

Primero, esimportanteprecisarqueel presente estudio tiene cobertura parcial, y no total para el país; los beneficiarios del proyecto o "grupo de tratamiento" se comparan con un grupo no beneficiario con similares características. Si este último grupo se selecciona de manera aleatoria, se le denomina "grupo de control"; y si es seleccionado de otra manera, se le denomina "grupo de comparación". La selección del "grupo de tratamiento" no es aleatoria, por lo que no se asume un tipo de diseño experimental sino cuasi experimental. Además, en los costos de este tipo de estudios, cuando la cobertura es completa, es decir, cuando se tiene un alcance nacional, la comparación es compleja y costosa, dado que debe recurrirse a la construcción de modelos computables de equilibrio general, comparación entre países, etc.

Sin embargo, en este estudio se ha realizado la evaluación del impacto del Programa Hábil
Técnico Permanente con cobertura nacional y se toma en cuenta para su evaluación respectiva al grupo de tratamiento, para observar su comportamiento tomando de referencia al que corresponde al grupo de no participantes. ${ }^{2}$ Para el caso del grupo de participantes del cual se levantó la información contemplada en la boleta de trabajo, y que se entrenó en los diferentes cursos de capacitación, lo conformaron jóvenes que no fueron definidos de manera aleatoria; por consiguiente, las características que asume este análisis es de carácter cuasi experimental.

En términos de procedimiento, selecciono un grupo de comparación a partir de la participación relativa del levantamiento realizado en los departamentos de todo el país. ${ }^{3}$ El grupo de comparación se realizó tomando en cuenta la participación relativa del levantamiento en cada municipio y por departamento. Estos datos muestrales se extrajeron de la Encuesta de Hogares de Propósitos Múltiples 2010, que levanta la Dirección General de Estadística y Censos, DIGESTYC, igual número para cada municipioy departamento, tomando en cuenta variables específicas de comparación como son sexo, estado familiar, edad, educación, ingreso, etc., con un nivel de confianza del 95\% de que ambas muestras son estadísticamente significativas y que poseen las características similares en esas variables.

Posteriormente, se aplicó la técnica “doble

2 Si la selección es aleatoria se estaría frente a un diseño experimental. Esto implica que desde el principio se deberá partir de un conjunto bien definido de personas para hacer la selección, tanto para el grupo donde el proyecto se realiza la intervención (grupo en tratamiento), como el de control, lo cual no es el caso de la presente consultoría.

3 Los departamentos en los cuales se levantó la información del grupo de tratamiento fueron Ahuachapán, Santa Ana, Sonsonate y Cabañas, a excepción de La Libertad, departamento en donde no se pudo realizar el levantamiento respectivo. 
diferencia" o "diferencia en la diferencia", es decir, midiendo los promedios de las muestras del grupo de tratamiento $y$ de comparación o pareo, con lo cual se han medido los impactos respectivos. En síntesis, de los registros que proporcionó INSAFORP se levantó la muestra teórica de 390 participantes de aquellos que fueron sujetos de apoyo con el Programa Hábil Técnico Permanente. ${ }^{4}$ Además, con la muestra del grupo de pareamiento se han realizado múltiples pruebas en dirección a poseer la consistencia estadística robusta requerida. En síntesis, este método a aplicar, que tiene una larga historia, es el método más común en la evaluación de impactos debido a que no necesita construcciones específicas, tal como se ha dicho más arriba.

\subsubsection{Definición y análisis de las variables}

La variable ocupación representa la condición laboral, es decir, si trabaja o no trabaja, variable dicotómica, es decir, si el participante es ocupado adopta el valor de uno, pero si no está ocupado adopta el valor de cero de ambos grupos muestrales. ${ }^{5} \mathrm{Se}$ espera una relación positiva entre la variable explicativa ocupación, variable dicotómica, y la variable ingresos que es la variable dependiente. Esto se explica, ya que en la medida en que exista condición de trabajo existirá un mayor ingreso, mejorando de esa manera el bienestar de los individuos. A través de esta relación se mide el impacto de la condición de empleabilidad lograda,

4 El marco muestral que se ocupará para seleccionar el grupo en tratamiento y el grupo control, son las personas egresadas del Programa Hábil Técnico Permanente para los años 2010-2011.

5 En el modelo que se aplica se utilizan datos muestrales para el grupo de tratamiento y grupo de control. posterior a los cursos de formación, sobre los ingresos del individuo.

Otra variable fundamental es negocio, y está referido a que el individuo posea un negocio; en este caso se espera un signo positivo para esa variable, que es una variable dicotómica, que toma el valor de uno si el individuo se ocupa y tiene un negocio, y de cero si no lo tienen. Esto es así, ya que la probabilidad de empleabilidad es mayor cuando se cuenta con un negocio propio.

También se considera la variable zona, ya que se espera que los jóvenes que se emplean provienen de la zona urbana, y esto es así por la red de amigos y de instituciones que pueden promover la empleabilidad en la zona rural. En ese sentido, la variable zona, que es una variable dicotómica, toma el valor de uno si el individuo vive en la zona urbana, y de cero si el individuo vive en una zona rural, y se espera que la relación entre esta variable y el ingreso posea signo positivo.

Teóricamente se sabe que el nivel de ingreso se incrementa si el individuo posee un nivel más alto de educación; por tanto se espera que el coeficiente de la variable $\sqrt{\text { EDUCACIÓN }}$ que es la raíz cuadrada de los años de educación del individuo, tenga signo positivo.

También otra variable de control es la edad, de la cual se espera que el signo del coeficiente sea positivo y haya una relación no lineal entre los ingresos y la edad del individuo; por tanto, se transforma en logaritmo. De hecho, a cierta edad los ingresos de los individuos empiezan a disminuir por diferentes 
motivos, tal como asegura el retiro $\mathrm{u}$ otros motivos.

La variable sexo del modelo de impacto es una variable dicotómica que toma uno si el individuo es hombre, y el valor de cero si el participante es mujer. No se puede decir de manera categórica que el coeficiente de esta variable tendrá un signo positivo o negativo porque hasta ahora no existe una teoría bien establecida, para el nicho de mercado en estudio sobre su comportamiento, básicamente cuando se analiza la participación de la mujer en los mercados de tipo informal a diferencia del mercado formal del trabajo.

En el modelo la variable $D_{i}$ es una variable binaria que toma el valor de uno si la persona fue participante en los cursos de capacitación y cero si el individuo no fue afectado, es decir, no participó en los cursos de capacitación. En este caso, dado que los efectos que se esperan de haber participado en los cursos de formación, es que se incremente su nivel de bienestar observado en sus ingresos personales y que exista un impacto positivo por ese hecho. En síntesis, se espera un coeficiente de la variable que captura en el modelo la diferencia entre los afectados por los cursos de capacitación y los que no tuvieron esa oportunidad.

Para capturar el efecto del tiempo se incorpora en el modelo la variable ti que también es una variable dummy ${ }^{6}$, que es igual a cero, cuando son datos antes de ocurrencia

\footnotetext{
6 La palabra Dummy se refiere a que en el modelo se incluirán variables que adoptan dos valores. Cuando se tiene la participación por ejemplo del sexo, si es hombre podrá adoptar el valor de uno, y si es mujer es cero. Por ello son variables binarias, dicotómicas o Dummy.
}

de los cursos de capacitación, es decir, antes de 2010 y que forman parte de la muestra de comparación, y es igual a uno cuando son datos recolectados por la muestra del grupo de tratamiento, individuos participantes de los cursos de capacitación. El signo del coeficiente de esta variable dependerá de la evolución de los ingresos entre ambas muestras. Entonces, puede ser positivo si los individuos alcanzaron un aumento de sus ingresos de manera significativa antes de 2010 por otros sucesos extraeconómicos y negativo si los ingresos posteriores a 2011 son inferiores a los obtenidos antes de 2010, hecho que es independiente de los cursos de formación. El producto de la variable $D_{i}$ y la variable ti $\left(D_{i}, t_{i}\right)$ es la variable que va a expresar el efecto o impacto de los cursos de capacitación en los ingresos de los individuos. Las consecuencias de los cursos de formación en los participantes producen efectos en el corto y el mediano plazo, eso quiere decir que para expresar en el modelo el impacto positivo de estos cursos de capitación sobre los ingresos, el coeficiente de la variable $\left(D_{i}, t_{i}\right)$ debería tener signo positivo.

\subsubsection{El modelo}

El modelo de regresión que se adopta se describe así:

$$
Y_{i t}=\alpha+\beta D_{i t}+\gamma_{t i}+\delta\left(D_{i t} t_{i}\right)+\rho X_{i t}+\mu_{i t}
$$

Donde:

- i representa el número de variables, y

- trepresenta para nuestro caso, el número de observaciones, es decir, $\mathrm{N}=780$ cifra para el grupo de tratamiento como al número del grupo de comparación. 
- De esa manera Xit es el vector de las variables explicativas, y

- Yit(ingresos delosindividuos)corresponde a la variable dependiente a considerar.

La variable $t$, que también es una variable dummy que es igual a cero, cuando son datos antes de ocurrencia de los no participantes a los cursos de capacitación, que provienen de la EHPM 2010 y es igual a uno si los datos de los participantes del PHTP corresponden a los años 2010-2011.

La variable $\left(D_{i}, t_{i}\right)$ es la que expresa el efecto o impacto de los cursos de capacitación en los ingresos de los individuos.

La $\mu_{i t}$ representa el término de la perturbación estocástica.

\subsubsection{Forma funcional del modelo}

En esta sección se proponen los factores que inciden y determinan los impactos en el bienestar de los individuos, es decir, los ingresos. Por consiguiente, se plantearán las variables que producen esos impactos respectivos. La importancia de este suceso es que esta información, desde el punto de vista estadístico, se podrá inferir para el marco muestral con el que se ha trabajado y definir la tendencia para el país. Utilizando el paquete SPSS v. 20 se corrió la forma funcional, la cual presenta la siguiente estructura:

$Y_{i t}=a+\rho_{1}$ Ocupado $_{i t}+\rho_{2}$ Negocio $_{i t}+$

$\rho_{3} \sqrt{ }$ Educación $+\rho_{4} \log ($ edad $)+\rho_{5} \operatorname{Sexo}_{i t}+\beta D_{i t}$

$+\gamma t_{i}+\delta\left(D_{i t} t_{i}\right)+\mu_{i t}$
Donde:

- Yit = Ingreso de los participantes del grupo de tratamiento y no participantes es decir, del grupo de comparación.

- Ocupado it = Individuos que si poseen un trabajo=1 y si no lo posee $=0$ del grupo de tratamiento y del grupo de comparación.

- Negocio it = Individuos que cuentan con un negocio $=1$ y si no $=0$ del grupo de tratamiento y del grupo de comparación.

- $\sqrt{\text { Educación }_{\mathrm{it}}}=$ Años de estudios realizados del grupo de tratamiento y del grupo de comparación.

- Logedadit = logaritmo de los años cumplidos por los participantes del grupo de tratamiento y del grupo de comparación.

- Sexoit = Los individuos que sean hombres $=1$ si son mujeres $=0$ del grupo de tratamiento y del grupo de comparación.

- Dit = Es la variable que indica que si el individuo recibió cursos de capacitación $=1 \mathrm{sino}=0$.

- $t \mathrm{t}=$ Indica que si $\mathrm{t}=1$ grupo de tratamiento y si $t=0$ significa que pertenecen a grupo de comparación.

- $(\mathrm{Di}, \mathrm{ti})=$ Indica el efecto o impacto aditivo de los cursos de capacitación.

En resumen, se espera que los coeficientes, es decir todos los parámetros tengan un signo positivo a excepción del que corresponde a sexo que podría adoptar positivo con algunas reservas. 


\subsection{Hipótesis general del modelo}

En el bienestar de los participantes en el Programa Hábil Técnico Permanente medido a través de los ingresos de los jóvenes, es determinado y explicado por la formación o entrenamiento recibido a través de cursos de capacitación realizados en los años 2010 y 2011 por INSAFORP y de otras variables ya descritas más arriba.

\subsection{Supuesto específico: la homocedasticidad}

El modelo clásico de regresión lineal para estimaciones de corte transversal, por lo general no está afectado porla autocorrelación de cada una de las perturbaciones, es decir que $E\left(\mu_{i} \mu_{i}\right)=0$ donde $\mathbf{i} \neq \mathbf{j}$. Aunque la correlación generalmente predomina en series de tiempo.

El modelo que se ha considerado para medir el nivel de bienestar y los impactos de la empleabilidad alcanzada y otras variables, se basa en el supuesto que la varianza de cada término de perturbación $\mu_{i t}$ es condicional a los valores escogidos de las variables explicativas, es un número constante igual a . $\sigma^{2}$ Este es el supuesto de homocedasticidad o igual (homo) dispersión (cedasticidad), es decir, igual varianza. En símbolos: $E\left(\sigma_{i}^{2}\right)=\sigma^{2} i=1,2, \ldots, N$

\section{Interpretación de resultados}

\subsection{Aplicación del modelo e interpretación de resultados}

Los resultados del modelo muestran una relación positiva entre el nivel de ocupación con impacto sobre la variable de ingresos con una relación positiva como lo esperado y es altamente significativa. Eso se explica en la medida en que se tenga y se acceda a un empleo, se tiene una probabilidad en términos promedio de incrementar el ingreso en 128.3 puntos de diferencia sobre aquellos que no gozan de esa oportunidad. Esto es el impacto del PHTP que permite y asegura reducir los niveles de pobreza, hipótesis de trabajo sobre la cual se ha trabajado a lo largo de este estudio.

La variable negocio con -47.4 indica que el signo no es el esperado, aunque es estadísticamente significativa. Se sugiere no considerarla en el modelo y esperar a revisar otros estudios sobre el comportamiento de esta variable.

La variable zona de residencia con un coeficiente de 12.4 indica que en efecto aquellos participantes del sector urbano poseen una potencialidad frente a los del área rural. El signo aparece como el que en efecto se esperaba. La variable no es estadísticamente significativa en más del $95 \%$ de confianza. Como la variable toma el valor de uno, si el participante habita en la zona urbana, y cero si habita en la zona rural, se espera que el impacto sea mayor cuando procede del área urbana.

La variable raíz cuadrada de la educación, que se esperaba una relación positiva no lineal entre el número de años de educación de los participantes, tanto del grupo de comparación como del grupo de tratamiento. El resultado es -21.6 con signo diferente al esperado, aunque es estadísticamente significativa.

La variable sexo se comporta con fuerte impacto pues, además de que el signo es el esperado es altamente significativa con 54.7 , lo que indica que efectivamente esta variable impacta cuando es hombre, 
frente al caso de ser mujer en los cursos de formación.

En relación a la variable Logedad, esta indica que el resultado es 116.9 y es positivo, y es altamente significativo. Por lo que existe una relación positiva y logarítmica entre la edad del participante y el ingreso que es la variable dependiente.

La variable $\mathrm{D}$ del individuo recibió cursos de capacitación tiene un impacto positivo sobre los ingresos promedios mensuales, y se encuentra con el signo esperado. Es, además, estadísticamente significativa. Esto indica que su coeficiente 169.1 es la diferencia de los participantes por encima y con respecto a los que no participaron en los cursos de formación impartidos por INSAFORP.

Finalmente, la incidencia conjunta de ambas variables $D$ y $t$ deben ser estudiadas más en detalle para valorar su introducción al modelo. El resultado indica signo negativo, aunque es estadísticamente significativa.

Variable dependiente Ingresos en US\$ promedios mensuales.

Método de mínimos cuadrados ordinarios. Muestra $=780$ participantes de grupo de tratamiento y grupo de comparación. Programa SPSS v.20.

\section{Cuadro 3}

El Salvador. Aplicación de modelo econométrico de grupo de tratamiento del PHTP y grupo de comparación de la EHPM.

\begin{tabular}{|c|c|c|c|c|c|}
\hline \multirow{2}{*}{ Modelo } & & \multicolumn{2}{|c|}{ Estandarizados } & \multirow{2}{*}{$\mathbf{t}$} & \multirow{2}{*}{ Sig. } \\
\hline & & & & & \\
\hline \multirow[t]{9}{*}{1} & (Constante) & -192.95 & $54 \cdot 32$ & -3.55 & 0.000 \\
\hline & OCU & 126.94 & 12.33 & 10.29 & 0.000 \\
\hline & NEG & -47.45 & 14.30 & -3.32 & 0.001 \\
\hline & ZON & 10.50 & 13.42 & 0.78 & 0.434 \\
\hline & RAIZ & -21.60 & 3.96 & -5.46 & 0.000 \\
\hline & Log edad & 116.97 & 35.68 & 3.28 & 0.001 \\
\hline & Sexo & 54.71 & 11.08 & 4.94 & 0.000 \\
\hline & $\mathrm{D}$ & 169.15 & 18.11 & $9 \cdot 34$ & 0.000 \\
\hline & DT & -0.14 & 0.07 & -2.13 & 0.033 \\
\hline
\end{tabular}

Fuente: Banco mundial, 2013 y elaboración propia. 


\section{Conclusiones}

- El Programa Hábil Técnico Permanente 2010-2011 tiene un fuerte impacto en la empleabilidad y en los ingresos de los participantes. La oferta recibida a través de los centros de formación profesional autorizados por INSAFORP tiene un fuerte impacto en las condiciones básicas para la empleabilidad de los participantes.

- Los impactos cuantitativos y cualitativos de los cursos impartidos generan un incremento del bienestar en los participantes. Los resultados de la evaluación de impacto de la oferta de cursos de formación generan empleabilidad y mejora de los ingresos en el marco del Programa Hábil Técnico Permanente $y$, en consecuencia, en la mejora de las condiciones de vida de la población participante, impactos positivos, logrando los objetivos del Programa frente aquellos que no lograron participar en los años 2010 y 2011.

- El Programa Hábil Técnico Permanente 2010-2011 posee trazabilidad de género. De nuevo se registra un dato sumamente importante en términos de los participantes. Las mujeres experimentan mayor propensión al logro de la empleabilidad, dado que las especialidades ofrecidas fueron mayormente aquellas que poseen un estigma que son propias para mujeres.

- La educación de base de los participantes requiere de más formación formal para elevar los niveles de productividad. Los jóvenes participantes manifestaron que las razones que obstaculizan para la contratación en el sector formal, en primer lugar, son las de que el empleador requiere de personal experimentado; en segundo lugar, los participantes consideran que muy poco pagan los empleadores; y en tercer lugar, manifestaron que el nivel de escolaridad era insuficiente como para ser contratado.

- Persisten limitantes para la adecuada aplicabilidad de los conocimientos adquiridos por los participantes. En la medición del impacto sobre los cursos recibidos sobre su aplicabilidad o no, en el empleo, los participantes tienen la opinión de que ha sido de mucha utilidad, tanto para el trabajo como para poner un negocio.

- La metodología utilizada para comparar los resultados entre el grupo de tratamiento y el de comparación posee un rigor analítico que genera confianza en la evaluación del Programa. La evaluación de impacto del Programa, comparando los resultados alcanzados por los egresados, respecto al grupo de comparación, muestra un impacto positivo en todas las variables evaluadas.

Artículo recibido: 15 de mayo 2014

Artículo aprobado: 27 de junio 2014

\section{Bibliografía}

ABDALA, E. Formación y empleabilidad de jóvenes en América Latina, en M. Molpeceres Pastor (coord.) Identidades y formación para el trabajo, Montevideo, organización Internacional del Trabajo (OIT)/Centro Interamericano de Investigación y 
Documentación sobre Formación Profesional (CINTERFORP), 2004.

BAKER, J. Evaluación del impacto de los proyectos de desarrollo en la pobreza, Manual para profesionales, Banco Mundial, Washington, D.C., 2000.

BROWN, A. Regression et Correlation, Press de l'Université Laval, Quebec, 1978.

DIGESTYC. Encuesta de Hogares de Propósitos Múltiples, varios años.

D. O. No. 143, Tomo No. 320 Instituto de Formación Profesional, INSAFORP, 1993.

DIGESTYC. Encuesta de Hogares de Propósitos Múltiples, (EHPM), 2012.

EVIEWS USER'S GUIDE, VERSION 3.0, QMS Quantitatve Micro Software Irvine, California.

FLACSO. Mapa de pobreza: Política social y focalización, 2005.

GALLART, MARÍA ANTONIA. La formación para el trabajo y los jóvenes en América Latina, Santiago de Chile, 2003.

GLEJBERMAN, DAVID. Curso de muestreo aplicado a encuestas económicas, DIGESTYC-2007.

GUJARATI, N, DAMODAR. Econometría, Segunda edición, Editorial, McGraw.

INSAFORP. Evaluación de impacto: incidencia de la capacitación para el trabajo en la incorporación al empleo, de la población egresada del programa habilitación para el trabajo, años 2001 y 2002.
INSAFORP. Evaluación de impacto: incidencia de la capacitación para el trabajo en la incorporación al empleo, de la población egresada del programa habilitación para el trabajo, años 2001 y 2002 (2013).

JOHNSTON, J., Methodes Econometriques, tome 1 et 2 3a. Edition, Economica, 1985.

MINISTERIO DE EDUCACIÓN, Plan Nacional 2021, Metas y Política para Construir el País que Queremos. Primera edición, marzo 2005.

NINA BILLOROU Y OTROS. Guía para la Evaluación de Impacto de la Formación, CINTERFOR-OIT, Montevideo, Uruguay, 2011, Pág. 20.

NÚÑEZ, SERGIO. "Evaluación de impacto de un programa de entrenamiento laboral a través de modelos econométricos. El Caso de Proyecto Joven. República de Argentina": Monografía de Graduación realizada como parte de los requerimientos para mantener el grado de master of Arts Economics, Georgetown University, Buenos Aires, mayo de 2002.

ORGANIZACIÓN INTERNACIONAL DEL TRABAJO, OIT. La OIT en América Latina, y el Caribe, Avances y perspectivas, 2013.

ORGANIZACIÓN MUNDIAL DEL TRABAJO, OIT, Tendencias mundiales del desempleo 2013.

\section{PROGRAMAS ESTADÍSTICOS: MINITRAB}

WELLER, JURGEN. La inserción laboral de los Jóvenes, características tensiones y desafíos, Revista de la CEPAL, No. 92, 2007. 
ORGANIZACIÓN INTERNACIONAL DEL TRABAJO. La crisis del empleo juvenil: Un llamado de acción, Resolución y conclusiones de la $101^{\mathrm{a}}$. Reunión de la conferencia Internacional del Trabajo, Ginebra, 2012.

OIT, Informe mundial sobre salarios 2012/ 2013, Los salarios y el crecimiento equitativo, Ginebra.

PNUD, Informe de Desarrollo Humano. El Salvador 2013, Imaginar un nuevo país. Hacerlo posible. p.351.

RANIERI, RAFAEL AND RAQUEL ALMEIDA. What is Inclusive Growth?, International Policy Centre for Inclusive Growth (IPC-IG).

\section{Artículos}

BID. Dimensión del mercado de remesas, marzo de 2007.

BID. ¿De tal palo, tal astilla?, noviembre de 2007.

FMI. La economía crecerá poco, La Prensa Gráfica, síntesis de la Agencia EFE, 2008.

MORENO, LUIS ALBERTO. Los de afuera, Presidente Banco Interamericano de Desarrollo, BID, octubre de 2007.

SEMINARIO RIAL SOBRE EMPLEO DE JÓVENES, Río de Janeiro, mayo 20 y 21, 2008.

GALLART, MARÍA ANTONIA. La formación para el trabajo y los jóvenes en América Latina, Santiago de Chile 2003, 40p.

\section{Sitios web}

http://www.sedi.oas.org/ddse/espanol/ cpo_trab_rial_jovenes.asp\#_Hlk3

http://unstats.un.org/unsd/mdg/Host. aspx?Content=Indicators/OfficialList.htm.

http://www.iadb.org/NEWS/articledetail.cf $\mathrm{m}$ ? Language $=$ Sp\&parid $=4 \&$ artType $=W S \& a r$ tid $=4377$

www.cepal.org, CEPAL, Notas de la CEPAL

www.cepal.org, Machinea, José Luis, CEPAL

http://www.sedi.oas.org/ddse/espanol/ cpo_trab_rial_jovenes.asp\#_Hlk3 